\title{
Detection Sensitivity and Temporal Resolution of Visual Signals near Absolute Threshold in the Salamander Retina
}

\author{
E. J. Chichilnisky ${ }^{1 \star}$ and F. Rieke ${ }^{2 \star}$ \\ ${ }^{1}$ Systems Neurobiology Laboratory, The Salk Institute, La Jolla, California 92037, and 2Department of Physiology and Biophysics, University of Washington, \\ Seattle, Washington 98195
}

\begin{abstract}
Several studies have suggested that the visual system can detect dim lights with a fidelity limited only by Poisson fluctuations in photon absorption and spontaneous activation of rhodopsin. If correct, this implies that neural processing of responses produced by rod photoreceptors is efficient and effectively noiseless. However, experimental uncertainty makes this conclusion tenuous. Furthermore, previous work provided no information about how accurately stimulus timing is represented. Here, the detection sensitivity and temporal resolution of salamander rods and retinal ganglion cells (RGCs) are compared in nearly matched experimental conditions by using recorded responses to identify the time of a flash. At detection threshold, RGCs could reliably signal the absorption of $20-50$ photons, but the rods within the RGC receptive field could signal stimuli 3-10 times weaker. For flash strengths 10 times higher than detection threshold, some RGCs could distinguish stimulus timing with a resolution finer than $100 \mathrm{msec}$, within a factor of 2 of the rod limit. The relationship between RGC and rod sensitivity could not be explained by added noise in the retinal circuitry but could be explained by a threshold acting after pooling of rod signals. Simulations of rod signals indicated that continuous noise, rather than spontaneous activation of rhodopsin or fluctuations in the single-photon response, limited temporal resolution. Thus, detection of dim lights was limited by retinal processing, but, at higher light levels, synaptic transmission, cellular integration of synaptic inputs, and spike generation in RGCs faithfully conveyed information about the time of photon absorption.
\end{abstract}

Key words: absolute threshold; signal processing; physical limits; visual sensitivity; photon detection; scotopic vision

\section{Introduction}

The elementary function of vision is to sense the arrival of photons at the retina. The visual system performs this task with an accuracy approaching the limit set by the division of light into discrete photons. Rod photoreceptors can faithfully signal the absorption of individual photons (Baylor et al., 1979b), and the amplification and low dark noise required for this acute sensitivity have begun to be understood (for review, see Rieke and Baylor, 1998b). Furthermore, physiological and behavioral studies indicate that downstream circuits can detect the absorption of a few photons in a pool of several hundred rods (Hecht et al., 1942; Barlow et al., 1971; Sakitt, 1972; Copenhagen et al., 1987), although less is known about the mechanisms responsible.

Previous work has led to the hypothesis that the main neural limit to visual sensitivity is physiological noise caused by spontaneous activation of rhodopsin in the rods (for review, see Field et al., 2005); this noise is indistinguishable from the signal produced by photon absorption. This hypothesis, if true, indicates that

\footnotetext{
Received June 15, 2004; revised Nov. 12, 2004; accepted Nov. 12, 2004.

This work was supported by National Eye Institute Grants EY13150 (E.J.C.) and EY11850 (F.R.), by the McKnight Foundation (E.J.C., F.R.), and by the Sloan Foundation (E.J.C.). We thank Peter Detwiler, Felice Dunn, Greg Field, and Valerie Uzzell for careful reading of this manuscript and Denis Baylor, Geoff Owen, and Fred Soo for insightful discussion and participating in initial experiments.

*E.J.C. and F.R. contributed equally to this work.

Correspondence should be addressed to E. J. Chichilnisky, Systems Neurobiology, The Salk Institute, 10010 North Torrey Pines Road, La Jolla, CA 92037. E-mail: ej@salk.edu.

DOI:10.1523/JNEUROSCI.2339-04.2005

Copyright $\odot 2005$ Society for Neuroscience $\quad$ 0270-6474/05/250318-13\$15.00/0
}

downstream processing of rod signals is efficient and nearly noiseless. In cat, the absorption of a single photon in a rod can elicit two to three spikes in a downstream retinal ganglion cell (RGC) (Barlow et al., 1971); similar spontaneous bursts of spikes occur in darkness and limit the fidelity of photon sensing (Barlow et al., 1971; Mastronarde, 1983a,b). The dark firing rate of cat RGCs is in rough agreement with predictions from spontaneous activation of rhodopsin in rods and anatomical estimates of the number of rods providing input to the RGC (Baylor et al., 1984; Freed and Sterling, 1988; Sterling et al., 1988). In toad, RGC sensitivity is within a factor of 2-3 of the limit set by spontaneous activation of rhodopsin (Copenhagen et al., 1987; Hemila et al., 1998), a finding supported by the temperature dependence of behavioral sensitivity (Aho et al., 1988) (but see Aho et al., 1993). Finally, the sensitivity of human observers (Hecht et al., 1942; Sakitt, 1972) is limited by neural noise whose magnitude is approximately comparable with the measured rate of spontaneous activation of rhodopsin in rods. However, behavioral studies involved significant experimental uncertainty about the number of photons reaching the retina and do not provide a unique estimate of the intrinsic neural noise (Barlow, 1977; Teich et al., 1982; Donner, 1992). Furthermore, physiological estimates of the rate of rhodopsin activation in mammalian rods are themselves subject to considerable uncertainty (Baylor et al., 1984).

Thus, it remains unclear whether visual sensitivity reaches the limits imposed by spontaneous activation of rhodopsin. Several issues need to be resolved. First, other known sources of noise in 
rods, such as continuous dark noise (Baylor et al., 1980; Rieke and Baylor, 1996) and variability in the single-photon response (Baylor et al., 1979b; Rieke and Baylor, 1998a), might be expected to interfere with faithful photon sensing. Understanding the relationship between rod noise and visual sensitivity requires consideration of all three rod noise sources, especially because continuous noise may play a more important role in some species than others (Baylor et al., 1980, 1984). Second, limitations in downstream processing of rod signals, such as channel noise, synaptic transfer, and spike generation, could limit the fidelity of photon sensing (Dhingra and Smith, 2004). Third, previous studies focused only on the ability to detect a dim light. Many visual tasks, such as motion detection, rely on determining the relative timing of stimuli and thus require extracting timing information from rod responses. Little is known about how accurately timing information is represented in the rod or RGC signals for stimuli near visual threshold, an important consideration for visually guided behavior.

Here we examine how faithfully information about the absorption of photons in salamander rods is conveyed through the retinal circuitry to RGCs. We compare discrimination performance based on rod and RGC responses to weak flashes in experimental conditions in which the number of absorbed photons can be measured accurately and flash timing influences discriminability. RGCs failed to detect the weakest stimuli encoded by the collection of rods from which they receive input, apparently because rod signals were suppressed by thresholding in the retina occurring after signals from different rods are pooled. However, for stimuli well above threshold, some RGCs encoded stimulus timing with a fidelity approaching the limit imposed by rod noise. In these conditions, continuous noise in rods, rather than spontaneous activation of rhodopsin or noise in downstream retinal circuits, imposed the primary limit on visual performance.

\section{Materials and Methods}

\section{Suction electrode recordings of rod light responses}

Suction electrodes were used to record outer segment currents of rod photoreceptors from larval tiger salamanders (Ambystoma tigrinum). Animals were obtained from Kons Scientific (Germantown, WI) or Charles Sullivan (Nashville, TN) and were housed and killed according to procedures approved by the Administrative Panel on Laboratory Animal Care at Stanford University (Stanford, CA), the University of Washington (Seattle, WA), and the Salk Institute (La Jolla, CA). After dark adaptation overnight, the animal was killed, and the retina was isolated under infrared illumination using infrared image converters. A piece of retina $\sim 1 \mathrm{~mm}^{2}$ was shredded with fine needles in a drop of Ringer's solution. The resulting suspension was allowed to settle to the bottom of a recording chamber on the stage of an inverted microscope equipped with an infrared viewing system. Isolated cells were continuously superfused with bicarbonate Ringer's solution during recording. The Ringer's solution contained $110 \mathrm{~mm} \mathrm{NaCl}, 2 \mathrm{~mm} \mathrm{KCl}, 30 \mathrm{~mm} \mathrm{NaHCO} 3,1$ or $2 \mathrm{~mm} \mathrm{CaCl}_{2}$, $1.6 \mathrm{mM} \mathrm{MgCl}_{2}$, and $10 \mathrm{~mm}$ glucose; $\mathrm{pH}$ was 7.4 when equilibrated with $5 \% \mathrm{CO}_{2}-95 \% \mathrm{O}_{2}$, and osmolarity was $270-275 \mathrm{mOsm}$. Temperature was $20-22^{\circ} \mathrm{C}$.

Light-sensitive currents were recorded by drawing the rod outer segment into a glass electrode cut and polished to an opening of 12-14 $\mu \mathrm{m}$ (Baylor et al., 1979a). The electrode was filled with HEPES Ringer's solution ( $28 \mathrm{~mm} \mathrm{NaHCO}_{3}$ replaced with 3 mm HEPES and $25 \mathrm{~mm} \mathrm{NaCl}, \mathrm{pH}$ 7.4). Current collected by the suction electrode was low-pass filtered at 20 $\mathrm{Hz}$ (eight-pole Bessel low pass) and digitized at $100 \mathrm{~Hz}$. Saturating and half-saturating flashes were delivered periodically to check for stability of dark current and response sensitivity. We recorded exclusively from red rods, which account for $>90 \%$ of rods in the salamander retina (Sherry et al., 1998) and thus provide the primary input at low light levels. A total of 15 rods were used in the analysis. Responses to 50-150 flashes were recorded from each rod at each of three to four flash strengths.

\section{Instrumental noise in rod recordings}

Accurate estimation of rod sensitivity required recordings that were limited by cellular rather than instrumental noise. The relative magnitudes of cellular and instrumental noise were tested by measuring the current fluctuations in darkness and in saturating light for each rod. Saturating light isolated instrumental noise by eliminating the transduction current (Baylor et al., 1980). Data were retained only from rods in which the total noise in darkness exceeded that in saturating light for temporal frequencies below $10 \mathrm{~Hz}$, indicating that these frequencies were dominated by cellular rather than instrumental noise. The rod light response was also dominated by temporal frequencies below $10 \mathrm{~Hz}$, suggesting that estimates of sensitivity were not altered by high-frequency instrumental noise. This was confirmed by limiting the frequency content of the rod responses and checking for an effect on sensitivity. Eliminating temporal frequencies above $5 \mathrm{~Hz}$ by digitally filtering the rod responses produced a $<2 \%$ change in detection sensitivity and temporal resolution (see below). Eliminating temporal frequencies below $2 \mathrm{~Hz}$ decreased sensitivity $>10$-fold. Together, these observations indicate that discrimination was mediated almost entirely by frequencies lower than $5 \mathrm{~Hz}$ and, thus, that instrumental noise did not substantially alter estimates of rod sensitivity.

\section{Multielectrode recordings of $R G C$ light responses}

Retinas isolated from dark-adapted salamanders (see above) were placed flat against a planar array of 61 extracellular microelectrodes that were used to record action potentials from RGCs (Meister et al., 1994; Chichilnisky and Baylor, 1999). The retina was superfused with bicarbonate Ringer's solution (see above). Spike times, peaks, and widths were digitized at $20 \mathrm{kHz}$ (Meister et al., 1994; Litke, 1999) and stored for off-line analysis. Spikes from different cells were segregated by manually selecting distinct clusters in scatter plots of spike height and width recorded on each electrode and verifying the presence of a refractory period in the spike trains from each cluster. To verify refractory periods, a contamination measure was defined as the rate of spikes observed in the period $0.5-1.0 \mathrm{msec}$ after recorded spikes divided by the overall rate. Only clusters with low contamination were retained: of 85 RGCs examined in five retinas, 81 exhibited $0 \%$ contamination and four exhibited contamination lower than $10 \%$. Spikes recorded on multiple electrodes were identified by temporal coincidence; only spikes from the electrode with the most clearly defined cluster were retained. Cells or segments of experiments that exhibited obvious firing instability were excluded. Cells for which receptive fields were poorly defined were excluded (see below). Only cells that exhibited discrimination performance of at least $85 \%$ at the highest flash strengths and time offsets tested (see Results) were examined. Responses to 100-160 flashes were recorded at each flash strength.

\section{$R G C$ receptive field measurements}

Comparing rod and RGC sensitivity required estimates of RGC spatial receptive fields so that the number of rods providing input to the RGC could be determined. Receptive fields were measured by stimulating the retina with spatiotemporal white noise (or random flicker) for 20-30 min (Chichilnisky and Kalmar, 2002). The stimulus was composed of a lattice of squares 60 or $120 \mu \mathrm{m}$ on a side, refreshing at $67 \mathrm{or} 120 \mathrm{~Hz}$. The intensity of each monitor phosphor in each square was selected randomly and independently from one of two values on every refresh; in some experiments, the intensity of all three phosphors covaried. Receptive fields were obtained by computing the average stimulus in the $1 \mathrm{sec}$ period preceding a spike [the spike-triggered average (STA)]. Assuming that firing is controlled by a linear summation of recent intensity perturbations within the receptive field, the STA provides an estimate of the spatial, temporal, and chromatic properties of the light response (Chichilnisky, 2001). To summarize receptive field spatial extent, STAs were fitted with a space-time-color separable function (Chichilnisky and Kalmar, 2002). The spatial profile of this function was a two-dimensional elliptical Gaussian with no antagonistic surround.

Receptive field measurements were obtained using stimuli with mean intensity between 20 and 2000 photoisomerizations per rod per second $\left(\mathrm{Rh}^{*} / \mathrm{rod} / \mathrm{sec}\right)$. In a subset of experiments, the spatial receptive field diameter (diameter of a circle with the same area as the 1 SD elliptical 
boundary of the Gaussian fit) was examined at several light levels. Receptive field diameters decreased by $\sim 10 \%$ when the mean light level was raised from 200 to $2000 \mathrm{Rh}^{\star} / \mathrm{rod} / \mathrm{sec}$ but changed little between 20 and $200 \mathrm{Rh}^{\star} / \mathrm{rod} / \mathrm{sec}$, suggesting that receptive fields measured at $200 \mathrm{Rh}^{\star} /$ $\mathrm{rod} / \mathrm{sec}$ and below reflected the functional receptive field at absolute threshold. For one data set (see Fig. $8 A$ ), receptive fields were only measured at $2000 \mathrm{Rh}^{\star} / \mathrm{rod} / \mathrm{sec}$; in this case, a $10 \%$ upward correction was applied to the estimated receptive field diameters.

\section{Light calibration and comparison of different preparations}

For 13 of the 15 rod experiments, $10 \mathrm{msec}$ flashes were delivered using a light-emitting diode (LED) with peak emission at $470 \mathrm{~nm}$ imaged onto a $580 \mu \mathrm{m}$ spot centered on the recorded cell. Calibrated photon flux was converted to equivalent photons at the wavelength at which rod sensitivity was maximal ( $520 \mathrm{~nm}$ ) using published measurements of salamander red rod spectral sensitivity (Makino et al., 1991) and the measured LED spectral emission profile. Stimuli for the other two rods were delivered from an optical bench (see below).

In four of five RGC experiments, light from a tungsten-halogen lamp was imaged onto a $1.3 \mathrm{~mm}$ diameter uniform spot on the retina. Electronically controlled shutters generated $10 \mathrm{msec}$ flashes. An interference filter limited the incident light to a $10 \mathrm{~nm}$ band (full-width at half-height) centered at $506 \mathrm{~nm}$. Calibrated photon flux was converted to equivalent photons at $520 \mathrm{~nm}$ using the center wavelength and published measurements of the rod spectral sensitivity (Makino et al., 1991). In one experiment (see Fig. 8D), 10 msec flashes were generated using an LED with peak emission at $575 \mathrm{~nm}$. Calibrated photon flux was converted to equivalent photons at $520 \mathrm{~nm}$ as for rod LED stimuli.

Flash strengths were specified in terms of photoisomerizations per rod $\left(\mathrm{Rh}^{\star} / \mathrm{rod}\right)$, rather than photons per unit area, because several factors could cause the effective collecting area of the rods to depend on the experimental preparation and recording conditions. We accounted for these factors by measuring the collecting area in each case as described below.

In rod recordings, the collecting area was estimated from trial-to-trial fluctuations in the response to a fixed-strength flash. Assuming that the response fluctuations were dominated by the Poisson statistics that govern photon absorption, the ratio of the square of the mean response to the variance provides an estimate of the mean number of absorbed photons (Baylor et al., 1979b). The estimated collecting area obtained from 15 rods was $17 \pm 2 \mu \mathrm{m}^{2}$ (mean \pm SEM), with a range across cells of $10-23 \mu \mathrm{m}^{2}$. These values are similar to estimates based on outer segment volume and rhodopsin concentration (Harosi, 1975). The collecting area estimated from each rod was used to convert photon density to $\mathrm{Rh}^{\star} /$ rod.

In RGC recordings, the average rod collecting area was estimated from an absorption measurement at the end of each experiment. An area of retina $50-100 \mu \mathrm{m}$ in diameter was illuminated with $506 \mathrm{~nm}$ light from above, and the transmitted light was measured with a photomultiplier mounted below the mostly transparent electrode array. The difference in transmission before and after bleaching the photopigment was dominated by absorption by rhodopsin. The average rod collecting area was $a=\varphi f / d$, where $f$ is the fractional increase in transmission after bleaching, $d$ is the density of rods estimated from images of the rod mosaic, and $\varphi=0.6$ is the quantum efficiency of photoisomerization (Dartnall, 1972). Estimated collecting areas were between 15 and $29 \mu \mathrm{m}^{2}$ in five retinas tested with the $506 \mathrm{~nm}$ stimulus and $14 \mu \mathrm{m}^{2}$ in one retina tested with the $575 \mathrm{~nm}$ stimulus. The collecting area estimated in each experiment was used to convert photon density to $\mathrm{Rh}^{\star} /$ rod. In one experiment (see Fig. $8 \mathrm{~A}$ ), the collecting area was not measured, and average value from three absorption measurements performed with the same batch of animals was used instead.

\section{Interexperiment variability}

The flash strength required for criterion performance in rods (see Fig. 3) differed by as much as a factor of 3 across cells, whereas the SD was $\sim 30 \%$ of the mean. No selection was made of the recorded rods other than stability and a dark current exceeding $40 \mathrm{pA}$. Selection of RGC recordings was based on an approximate assessment of absolute sensitivity during the experiment. The entire experiment protocol was performed in a total of five preparations (see Fig. 8). In a similar number of experiments, the preparation was abandoned because of low sensitivity (at least five times less than the experiments retained). This selection was deemed necessary because the RGC sensitivity appeared to vary substantially more than that of the rods.

The Ringer's solution in initial experiments had a $\mathrm{Ca}^{2+}$ concentration of $1 \mathrm{~mm}$. In later experiments, the $\mathrm{Ca}^{2+}$ concentration was increased to 2 mM to reduce oscillations in RGC activity. Rod limit contours (see Fig. 8) were determined from rod measurements at both $\mathrm{Ca}^{2+}$ concentrations, and RGC sensitivity was compared with the relevant rod limit. Figure 8 , $A$ and $B$, was from experiments at $1 \mathrm{~mm} \mathrm{Ca}^{2+}$.

\section{Two-alternative forced-choice analysis}

The measures of rod and RGC stimulus discrimination described in Results relied on a simple procedure for inferring the time of the stimulus from the response. The response in each trial was summarized by a response vector, which specified the outer segment current as a function of time for rods (sampled in $0.01 \mathrm{sec}$ bins) and spike count as a function of time for RGCs (in bins of size $0.0125 \mathrm{sec}$; see below). A discriminant vector was created from the difference between the mean responses to two flashes delivered at different times (see Fig. 2 A). Identification of the stimulus from an individual response not used in calculating the discriminant was based on the correlation (inner product) of the response vector with the discriminant vector: positive correlation indicated an early flash time, and negative correlation indicated a late flash time (see Fig. $2 B$ ). Performance was measured as the fraction of trials in which this procedure correctly identified the time (early or late) of the stimulus. Below, we verify that the discrimination procedure used was not substantially biased and was nearly optimal.

Fits to discrimination surfaces, interpolated contours, detection, and timing thresholds

Discrimination performance was measured across a range of flash strengths and time offsets. This section introduces measures to summarize performance across these parameters. These measures are used in control analyses below.

Discrimination surfaces indicating performance as a function of flash strength and time offset (see Results and Figs. 3, 5) were fitted with a smooth functional form in which performance depends on an underlying signal-to-noise ratio. The signal-to-noise ratio was written as a product of terms involving the flash strength and time offset:

$$
\mathrm{SNR}=\mathrm{SNR}_{\max }\left[1-\exp \left(-\alpha \phi^{n}\right)\right][1-\exp (-\beta \Delta T)] .
$$

Here, $\phi$ is the flash strength, $\Delta T$ is the time offset, and $\mathrm{SNR}_{\max }, \alpha, \beta$, and $n$ are free parameters fitted to the data. $\mathrm{SNR}_{\max }$ indicates the signal-tonoise ratio for asymptotically large flash strengths and time offsets. The second term on the right side represents the contribution of the flash strength to the signal-to-noise ratio, and the third term represents the contribution of the time offset. Finally, model discrimination performance (probability correct) depends on SNR according to the following relation:

$$
P_{c}=\frac{1}{\sqrt{2 \pi}} \int_{-\infty}^{\mathrm{SNR}} e^{-x^{2} / 2} d x .
$$

This relation is expected for signals with independent and additive Gaussian noise with the given signal-to-noise ratio. Although this is at best an approximation of the statistics of retinal signals, Equations 1 and 2 were used only to obtain smooth fits to the discrimination surfaces and interpolate between measured points. Given fitted values of the parameters $\mathrm{SNR}_{\max }, \alpha, \beta$, and $n$, discrimination contours (see Fig. $3 D$ ) were obtained by setting SNR $=1$ and solving for values of $\phi$ and $\Delta T$ that satisfied Equation 1.

To test for systematic errors in the fits to rod discrimination surfaces, the differences between the fit and data were averaged across cells. All values in the resulting error surface were lower than $3 \%$. Similar averaging was not performed with RGCs, because discrimination surfaces for different RGCs had very different shapes. However, the root mean square 
Table 1. Control analyses

\begin{tabular}{|c|c|c|c|c|c|c|c|}
\hline \multirow[b]{2}{*}{ Cells } & \multirow[b]{2}{*}{ Analysis } & \multicolumn{3}{|c|}{ Detection } & \multicolumn{3}{|c|}{ Timing } \\
\hline & & $50 \%$ & $10 \%$ & $90 \%$ & $50 \%$ & $10 \%$ & $90 \%$ \\
\hline \multirow[t]{4}{*}{ Rods } & Subsample half & 1.02 & 0.91 & 1.12 & 1.04 & 0.90 & 1.18 \\
\hline & Variance normalization & 0.94 & 0.89 & 0.99 & 0.96 & 0.82 & 1.09 \\
\hline & Covariance normalization & 1.04 & 0.99 & 1.09 & 0.94 & 0.80 & 1.07 \\
\hline & Euclidean clustering & 1.05 & 0.95 & 1.15 & 1.08 & 0.89 & 1.27 \\
\hline \multirow[t]{5}{*}{$\mathrm{RGCS}$} & Subsample half & 0.99 & 0.89 & 1.08 & 1.04 & 0.89 & 1.29 \\
\hline & Variance normalization & 1.02 & 0.94 & 1.12 & 1.07 & 0.84 & 1.30 \\
\hline & Covariance normalization & 1.02 & 0.97 & 1.08 & 1.16 & 0.96 & 1.50 \\
\hline & Euclidean clustering & 1.01 & 0.96 & 1.05 & 1.00 & 0.93 & 1.10 \\
\hline & Non-Euclidean clustering & 0.99 & 0.90 & 1.05 & 0.89 & 0.70 & 1.07 \\
\hline
\end{tabular}

For each alternative analysis procedure, the detection and timing thresholds obtained using the alternative procedure were divided by those obtained using the standard procedure. The table shows the 50 th, 10 th, and 90 th percentiles of these ratios across all cells.

error between the fit and the data were between 1.5 and 5\% for all recorded RGCs (mean of 3\%).

For the control analyses described below, detection and timing thresholds were estimated from fits to discrimination surfaces. The detection threshold was defined as the flash strength that yielded SNR $=1$ for asymptotically long time offsets and was obtained by setting the third term in Equation 1 to unity and solving for $\phi$. The timing threshold was (arbitrarily) defined as the time offset that yielded SNR $=1$ for the highest flash strength tested and was obtained by solving Equation 1 for $\Delta T$ at this flash strength. These two measures constrain discrimination surfaces at the extremes of the flash strengths and time offsets tested.

\section{Finite data effects and temporal filtering}

The limited number of measured responses could underestimate the discrimination capacity of rods or RGCs if the waveform of the discriminant was strongly influenced by response noise and hence was not optimal. Such finite data effects are a particular concern for the discrete RGC responses: although a fine-grained temporal representation of spike trains (small time bins) could preserve the most detail in spike trains, a coarser representation (large time bins) could improve discrimination performance in a finite data set by reducing the impact of spike timing jitter. Thus, discrimination performance for RGCs was examined as a function of time bin size. For each time bin size, RGC detection and timing thresholds were computed as described above. To avoid the artifacts associated with arbitrary time bin boundaries, binned responses were filtered by circular convolution with a Gaussian filter, $f(t)=$ $\exp \left(-t^{2} / 2 \tau^{2}\right)$, with a value of $\tau$ equal to four times the time bin size. A filter width $\tau=0.05 \mathrm{sec}$ (and time bin size of $0.0125 \mathrm{sec}$ ) simultaneously minimized detection and timing thresholds for all discrimination procedures tested (see below). Similar temporal filtering did not reduce discrimination thresholds of rods.

To test for finite data effects, discriminability was examined in subsampled data. For both rods and RGCs, the number of trials was reduced by a factor of 2 , and the detection and timing thresholds obtained with subsampled data were divided by thresholds obtained with all data. The results in Table 1 show only a small effect of subsampling, indicating that finite data effects did not have a substantial impact on discrimination using rod or RGC responses, especially when compared with the 2- to 10-fold differences between RGC sensitivity and the limit set by the rods within the RGC receptive field (see Fig. 8).

\section{Adequacy of discrimination procedure}

The discriminant formed from the difference between the mean response vectors (see above) should yield optimal performance for discrimination of vectors with independent, equal variance noise in all entries (i.e., time points). Because this is at best an approximation to the statistics of rod and RGC responses, the discrimination procedure could underestimate the actual sensitivity of neuronal signals. To test for this possibility, several discrimination paradigms appropriate for more complex response statistics were tested. These produced at most small changes in performance compared with the differences between RGC performance and the rod limit; results are shown in Table 1.

Variance normalization. Different response variance in different time bins could result in suboptimal discrimination performance using the standard discriminant. This can be corrected by dividing the value of the discriminant in each time bin by the average variance of the early and late responses in that time bin. Variance normalization is the optimal procedure for discriminating two Gaussian distributions with equal, diagonal covariance (Duda and Hart, 1973).

Covariance normalization. Covariance in the values of different response time bins across trials could result in suboptimal performance using the standard discriminant. This can be corrected by normalizing the discriminant by the response covariance (Fisher discriminant); this is the optimal procedure for discriminating two Gaussian distributions with equal covariance (Duda and Hart, 1973). Because the dimension of the covariance matrix was high compared with the number of responses measured, the dimension of the data was reduced before computing the discriminant by projecting each response vector onto the first $d$ principal components of the pooled early and late responses. The Fisher discriminant was then computed by multiplying the standard discriminant by the inverse of the average of the covariance matrices obtained from responses to early and late stimuli (Duda and Hart, 1973). A value of $d=16$ was selected that simultaneously minimized detection and timing thresholds for both rods and RGCs.

Euclidean local clustering. The discrimination procedures above all rely on a discriminant computed in a way that weights all responses equally. These procedures cannot exploit local structure in response distributions. To test whether such a structure could be exploited by unequal weighting to improve discrimination, a local clustering procedure was applied (Duda and Hart, 1973; Victor and Purpura, 1997). For each response vector, the Euclidean distance between it and all other early and late response vectors recorded was computed, and each distance value was raised to a power $-p$. The sum of these values for the early and late responses was computed, and the stimulus was classified according to the response group with the smallest sum. For $p>0$, discrimination is dominated by the response vectors that are closest (in Euclidean distance) to the response being classified. A value of $p=1$ (i.e., vectors weighted by inverse Euclidean distance) simultaneously minimized detection and timing thresholds for rods and RGCs.

Non-Euclidean local clustering. For RGC spike trains, the Euclidean distance metric is complicated by the discretization of response vectors associated with binning spike times. An alternative, continuous measure of distance between spike trains is the total cost of transforming one spike train into another, using the elementary operations of shifting, adding, or deleting spikes (Victor and Purpura, 1997). The time scale $\tau$ of this metric is the inverse of the cost per unit time associated with shifting spikes, in which the cost of adding or deleting spikes is 1 . The local clustering procedure was applied to RGC responses using this distance metric instead of the Euclidean distance. A time scale parameter of $\tau=0.1 \mathrm{sec}$ and exponent of $p=1$ simultaneously minimized the detection and timing thresholds for RGCs.

\section{Model for rod pool signal}

The parameters of the model described in Equation 3 were estimated by fitting measured rod responses. Independent estimation of several parameters improved the accuracy of this procedure. First, $\bar{n}$ and $\sigma_{\mathrm{d}}$ were measured independently and held fixed. $\bar{n}$ was determined from the estimated rod collecting area and the photon density, and $\sigma_{\mathrm{d}}$ was determined from current records measured in darkness and analyzed identically to the flash responses. Second, distributions measured at four to five flash strengths were fitted simultaneously to find a common $\bar{c}$ and $\sigma_{c}$. This procedure was repeated for each time shift. Model parameters were combined across rods to estimate average rod behavior for each time shift. Parameters $\sigma_{\mathrm{c}}, \sigma_{\mathrm{d}}$, and $\bar{c}$ were first divided by the mean singlephoton correlation $\bar{c}$ and then averaged across cells. These averaged parameters were used to compute the rod limits to RGC sensitivity. 
Because each RGC receives input from hundreds of rods, the flash strengths used to elicit near-threshold responses from individual rods and RGCs differed by a factor of $10-30$. To determine the sensitivity limits of the pool of rods providing input to an RGC, the distribution of discrimination signals was fitted with the model of Equation 3, and the model was used to simulate responses of many rods at lower flash strengths (see Results). This procedure could introduce two kinds of systematic errors.

First, the fitting procedure could misestimate the magnitude of the signal relative to noise, and this error could be amplified when extrapolated to low light levels. To test for this, the fitting and extrapolation procedure was repeated using a known distribution of the form given in Equation 3. Random samples were drawn from this distribution. When Equation 3 was fitted to these samples, the estimated parameters differed from the original ones because of the finite number of samples. To test whether differences between the estimated and true distributions introduced systematic errors, samples were drawn from both distributions using lower flash strengths, and discrimination contours were computed. As shown in Figure $6 D$, this procedure did not reveal any systematic biases.

Second, the form of Equation 3 might differ from the true distribution of rod signals. For example, a Gaussian distribution of singlephoton response amplitudes provides a reasonable fit to the data but could be incorrect in detail. Such a discrepancy could be exacerbated when extrapolating to low light levels. A complete test of this possibility requires data that are difficult to obtain. However, a limited test was obtained by combining the measured responses to a dim flash with an appropriate number of sections of recordings in darkness to approximate the distribution of absorptions expected for flashes producing an average of $0.1-0.3 \mathrm{Rh}^{*}$. Discrimination performance using these resampled responses was compared with that of the model fit to the original data and extrapolated to the lower flash strength. Inadequacies in the form of the model should cause systematic differences between performance based on the resampled data and the model. No such differences were observed; in each case, the probability of correct stimulus identification based on the resampled responses was within $0.5 \%$ of that from the model fits.

\section{Results}

To determine whether rod noise or retinal processing limits visual detection sensitivity and timing resolution, we compared the fidelity with which rod photoreceptors and RGCs encoded weak visual stimuli. We focused on a simple discrimination task in which individual rod and RGC responses were used to infer the time of occurrence (early or late) of a brief flash. This simple task permitted identification of optimal or near-optimal procedures for using the neural response to discriminate stimuli, i.e., procedures with performance near that of an ideal observer. These discrimination procedures in turn permitted a direct comparison of rod and RGC sensitivity with minimal untested assumptions about how the stimuli are represented in the retina.

The rod-RGC comparison is presented below in four steps: (1) description of the discrimination task and characterization of the sensitivity of single rods; (2) characterization of RGC sensitivity; (3) characterization of the sensitivity of the collection of rods providing input to a single RGC; and (4) comparison with RGC sensitivity. $\phi=0.9 \mathrm{Rh}^{*}$

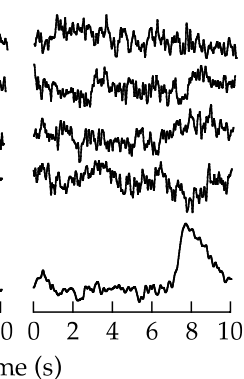

time (s)

D.
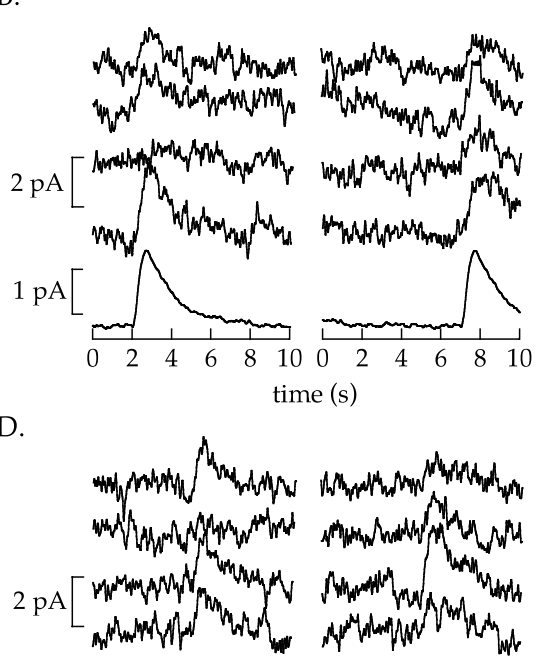

time (s)
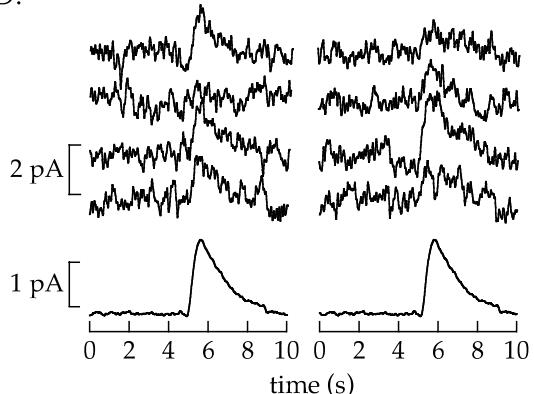

time (s)

$\phi=3.6 \mathrm{Rh}^{*}$

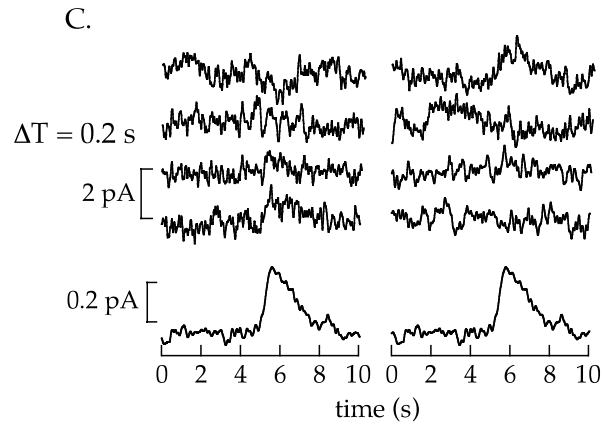

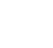

and temporal resolution. $A$, Responses to a flash producing an average of $0.9 \mathrm{Rh}^{*}$ at either $2 \mathrm{sec}$ (left) or $7 \mathrm{sec}$ (right). Average responses to 145 trials are shown at the bottom. Responses at 2 and $7 \mathrm{sec}$ were obtained by circularly time shifting the same data; hence, the average traces are time shifted copies of one another. The first 10 sec of the 12 -sec-long responses are plotted. B, Responses to a flash producing $3.6 \mathrm{Rh}^{*}$ at either $2 \mathrm{sec}$ (left) or $7 \mathrm{sec}$ (right). C, Responses to a flash producing $0.9 \mathrm{Rh}^{*}$ at either $4.9 \mathrm{sec}$ (left) or $5.1 \mathrm{sec}$ (right). D, Responses to a flash producing $3.6 \mathrm{Rh}^{*}$ at either

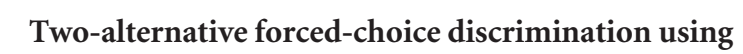
rod signals

To characterize rod sensitivity, dark-adapted rods were stimulated with brief, spatially uniform flashes. Examples are shown in Figure 1. Figure $1 B$ shows eight responses of a single salamander rod to flashes producing an average of $3.6 \mathrm{Rh}^{*}$. The left column shows four responses to flashes occurring at time $2 \mathrm{sec}$ (early), and the right shows four responses to flashes occurring at time $7 \mathrm{sec}$ (late). The last row shows average responses to flashes at the two times. The discrimination task was to classify the stimulus in each trial as early or late, using the recorded rod response. In this case, responses were easily detectable and the separation between the flash times was long, so the flash time could be identified easily in most trials.

The ease with which the flash time could be determined depended on both the flash strength and time offset. Figure $1 \mathrm{~A}$ shows responses obtained with weaker flashes $\left(0.9 \mathrm{Rh}^{\star}\right)$ and the same time offset $(5 \mathrm{sec})$. Although the flashes were widely separated in time, in most trials, a combination of rod current noise and Poisson fluctuations in the number of absorbed photons made it difficult to identify the response and thus determine whether the flash occurred early or late. Figure $1 D$ shows responses obtained with strong flashes $\left(3.6 \mathrm{Rh}^{\star}\right)$ offset in time by a smaller amount $(0.2 \mathrm{sec})$. Although individual responses were easily detectable, the small temporal offset between early and late responses was obscured by noise, making the task difficult. Figure $1 C$ shows responses obtained with weak flashes $\left(0.9 \mathrm{Rh}^{*}\right)$ and a short time offset $(0.2 \mathrm{sec})$; here, discriminating early flashes from late flashes based on individual responses is yet more difficult.

Rod detection sensitivity and timing resolution were quantified by measuring how accurately the time of the flash could be determined from the ensuing response. Flash responses, such as those shown in Figure 1, were recorded in 15 rods using five flash 
A.

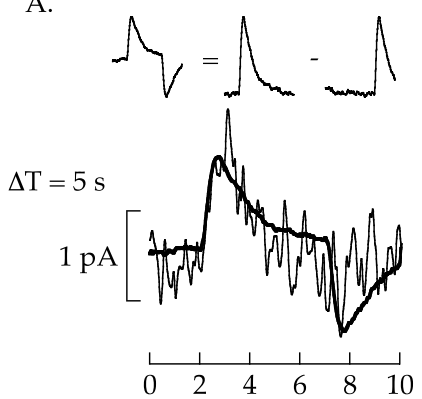

B.

$$
\phi=0.9 \mathrm{Rh}^{*}
$$

$\phi=3.6 \mathrm{Rh}^{*}$
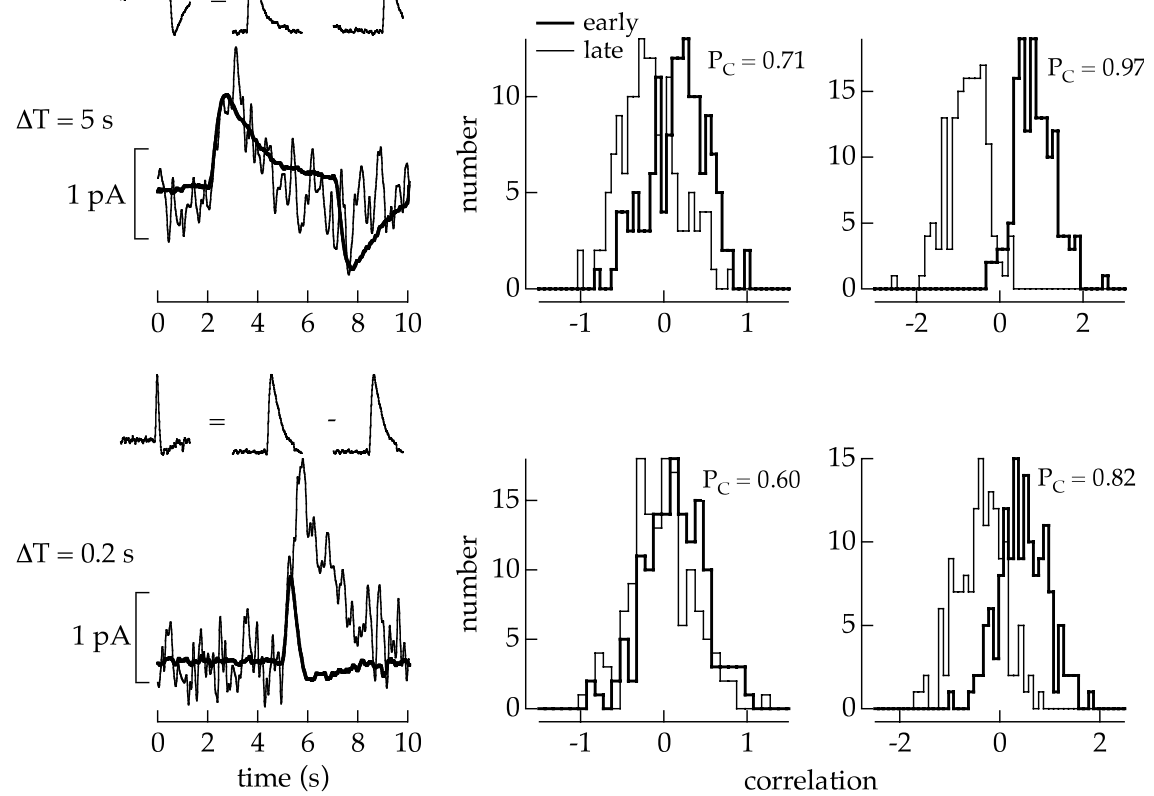
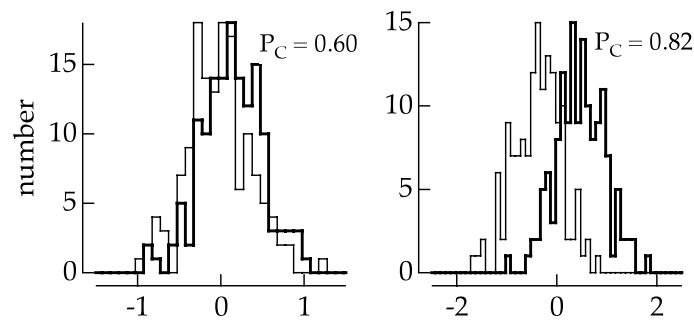

Figure 2. Early-late discrimination with rod responses. $A$, Discriminants (thick traces) formed by subtracting average late response from average early response and example individual responses for flashes separated by $5 \mathrm{sec}$ (top) and $0.2 \mathrm{sec}$ (bottom). $B$, Distribution of correlations for flashes producing an average of $0.9 \mathrm{Rh}^{*}$ (left) or $3.6 \mathrm{Rh}^{*}$ (right). Distributions are shown for flashes separated by $5 \mathrm{sec}$ (top) and $0.2 \mathrm{sec}$ (bottom). Same cell as Figure 1.

A

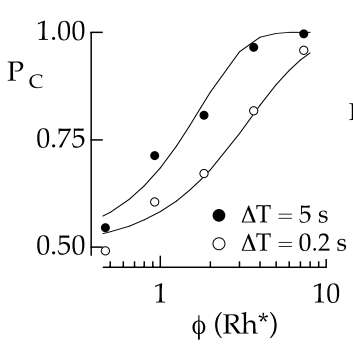

.

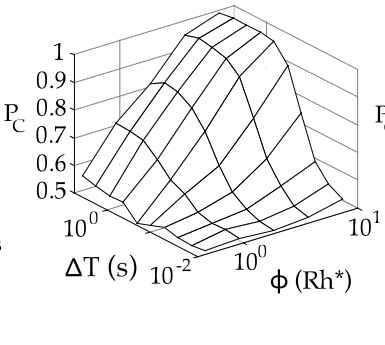

E.

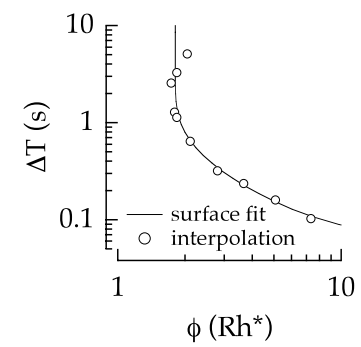

C.

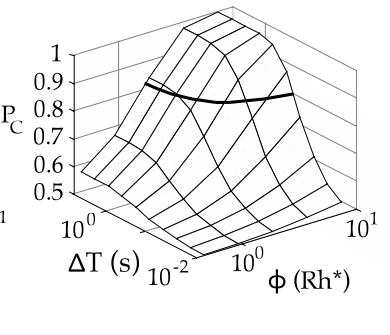

F.

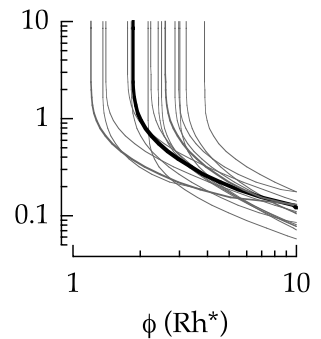

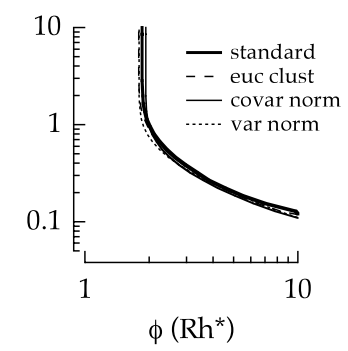

Figure 3. Rod discrimination performance across flash strengths $(\phi)$ and time offsets $(\Delta T)$. $A$, Discrimination performance plotted against flash strength for time offsets of $5 \mathrm{sec}()_{)}$and $0.2 \mathrm{sec}(\bigcirc)$. $B$, Discrimination surface summarizing performance across flash strengths and time offsets. C, Fit to discrimination surface computed according to Equations 1 and 2 . The thick line indicates the contour corresponding to $S N R=1 . D$, Comparison of discrimination contours from surface fit (line) and interpolated linearly from measured surface (O). E, Discrimination contours for 15 different rods (thin lines) and mean across cells (thick line). $F$, Comparison of discrimination contours for several alternative discrimination procedures (see Materials and Methods). standard, Standard procedure; euc clust, Euclidean clustering; covar norm, covariance normalization; var norm, variance normalization.

strengths. From each recorded response, a response to an "early" stimulus and a response to a "late" stimulus were extracted by circularly time shifting the same data. For each condition (e.g., flash strength of $3.6 \mathrm{Rh}^{\star}$, flash separation of $5 \mathrm{sec}$ ), a discriminant waveform was computed by subtracting the average late response from the average early response. Examples are shown for large and small time offsets in Figure $2 A$. The discriminant represents the information obtained over many trials about how responses to the early and late stimuli differ. The stimulus time (early or late) for a single response excluded from the discriminant calculation was inferred by computing the correlation, or inner product, of that response with the discriminant. A positive (negative) correlation indicates that the response was more similar to the average early (late) response. Figure $2 B$ shows, for each condition examined in Figure 1, distributions of the correlation values across trials. In each case, the separation of the distributions corresponding to early and late stimuli, which determines how accurately the two can be distinguished, confirms the qualitative impression of the difficulty of the task as a function of flash strength and time offset (Fig. 1).

Discrimination performance was defined as the fraction of trials in which the procedure correctly identified the time of the stimulus: that is, the probability of correct discrimination. The insets in Figure $2 B$ indicate the performance in each case. Because an equal number of early and late responses were used in the analysis, chance performance is 0.5 .

To characterize the fidelity of rod signals, discrimination performance was measured over a range of flash strengths and time offsets. Figure $3 A$ shows performance as a function of flash strength for the two time offsets examined in Figure 1. As expected, performance increased with flash strength, and achieving a given performance at the shorter time offset required higher flash strengths. These trends are summarized in the surface in Figure $3 B$, which shows discrimination performance for the same rod at all flash strengths and time offsets tested. The surface was approximated with the smooth function calculated according to Equation 1 and shown in Figure 3C (see Materials and Methods). The smooth lines in Figure $3 A$ show slices through the fit for time offsets of 0.2 and $5 \mathrm{sec}$, indicating an accurate functional approximation to the measured surface.

To summarize rod detection and timing sensitivity, the fit to the surface was used to estimate all of the combinations of flash strength and time offset that would result in a probability correct of 0.84 ( $\mathrm{SNR}=1$; see Materials and Methods). This is shown as a contour superimposed on the fit to the discrimination surface in Figure $3 C$. Figure $3 D$ shows that the contour lies near values obtained by linearly interpolating between points in Figure $3 B$, again confirming the functional approximation to the surface.

The discrimination contour will be used to compare the per- 
formance of different cells, discrimination procedures, and candidate limits to discrimination in the retinal circuitry. For long time offsets, performance depends only on the flash strength (i.e., is detection limited as in Fig. $1 A, B$ ), and the discrimination contour becomes nearly vertical. For short time offsets, performance is sensitive to both flash timing and strength (as in Fig. $1 C, D$ ), and the discrimination contour is angled. Figure $3 E$ shows discrimination contours from 15 rods (thin lines) and the mean across cells (thick line). In what follows, the mean rod is assumed to represent each of the rods providing input to an RGC.

Discrimination performance may be interpreted as a valid summary of rod signal fidelity only if the discrimination procedure extracts essentially all of the information present in the rod signals about the stimulus. This was tested by comparing the performance of the discrimination procedure with that of three more elaborate procedures that could, in principle, exploit more of the statistical structure of the rod signals (see Materials and Methods). The alternate procedures yielded very similar results (Fig. $3 F$ ), suggesting that the standard procedure accurately captured the detection and timing fidelity of rod signals.

\section{Two-alternative forced-choice} discrimination using RGC signals

RGC detection and timing sensitivity was determined using the same approach as that for rods (Figs. 1-3). Figure 4 shows sample responses of a single salamander RGC to early and late flashes at two flash strengths, in a similar format as the rod data of Figure 1. Early and late responses were obtained by circularly time shifting each measured response. Discrimination performance was measured by using each individual RGC response to classify the stimulus as early or late. As for rod responses, discrimination performance depended on both the flash strength and time offset. However, RGCs exhibited significant response nonlinearities. First, the response amplitude was not proportional to flash strength, e.g., the flash strength for Figure $4 B$ was approximately twice the flash strength for Figure $4 A$, but the mean response amplitude was approximately three times larger. Second, the response time course was generally not invariant with flash strength, e.g., the suppression of firing occurring 1-2 sec after the flash in Figure $4 B$ is not evident in Figure $4 A$. The latter observation implies that optimal stimulus discrimination based on RGC signals must use a different discriminant for every flash strength.

Figure 5 shows, in the same format as Figure 3, RGC discrim-

spike

$(\mathrm{Hz})$

spike

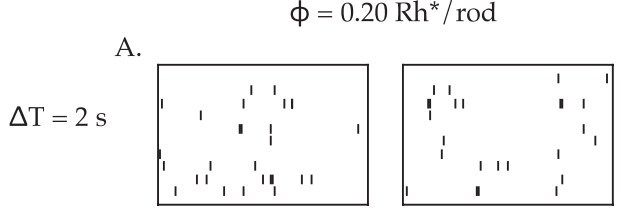

rate 2

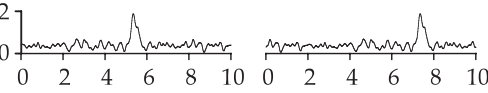

B.
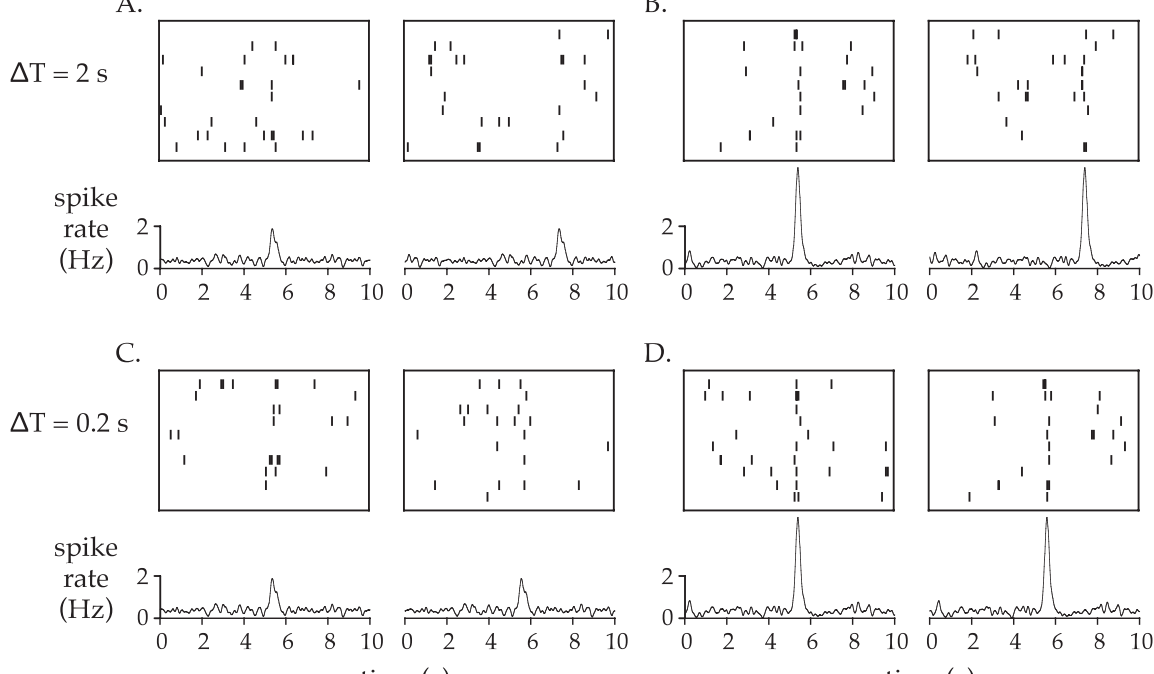

D.

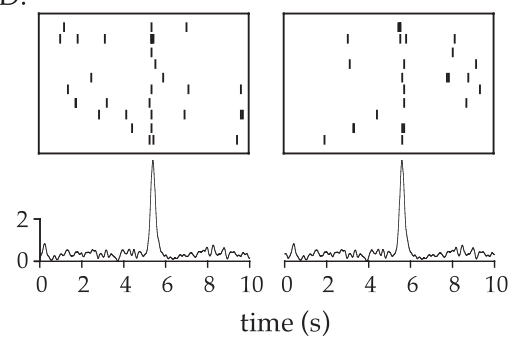

Figure 4. Task used to characterize RGC detection sensitivity and temporal resolution (see Fig. 1). Responses are shown for time shifts of $2 \mathrm{sec}$ (top) and $0.2 \mathrm{sec}$ (bottom) at flash strengths of $0.20 \mathrm{Rh}^{*} / \mathrm{rod}$ (left) and $0.39 \mathrm{Rh} / \mathrm{rod}$ (right). Ten individual responses are shown for the early and late stimuli for each flash strength and time shift combination. Average responses below the individual rasters were calculated across 160 trials. Responses at different times were obtained by time shifting the same data; hence, the average traces are time shifted copies of one another.
A.

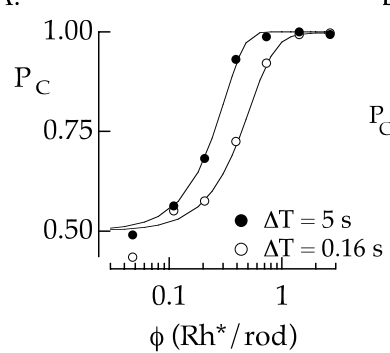

D.

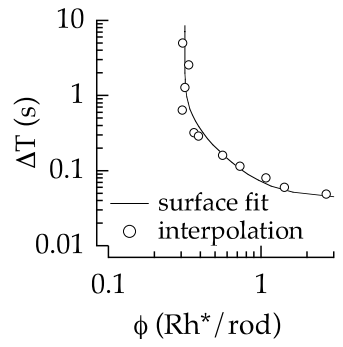

B.

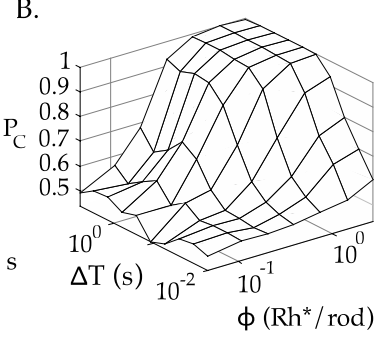

E.

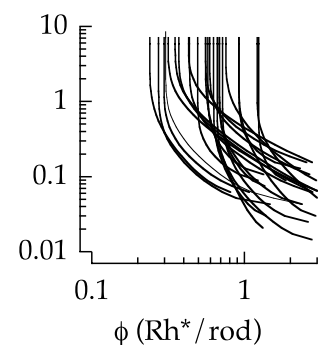

C.

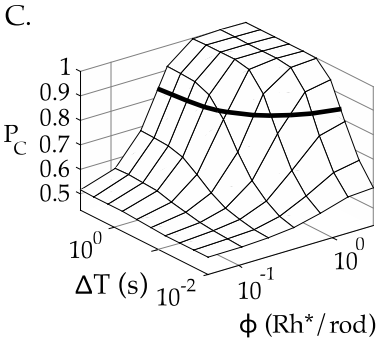

F.

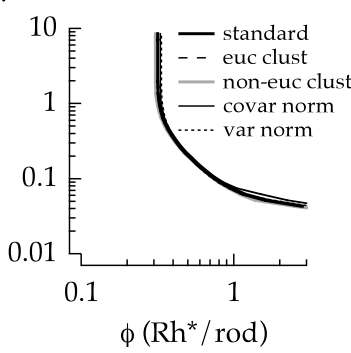

Figure 5. RGC discrimination performance across flash strengths $(\phi)$ and time offsets $(\Delta T)$ (see Fig. 3). $A$, Probability correct plotted against flash strength for large and small time offsets. $B$, Surface summarizing discrimination performance across a range of flash strengths and time offsets. C, Fit to discrimination surface from Equations 1 and 2. D, Discrimination contour (SNR $=1$ ) interpolated from the measured surface in $B(O)$ and calculated from the surface fit in $C$ (smooth line). E, Discrimination contours from 21 RGCs recorded simultaneously. F, Discrimination contours for a single RGC using several discrimination procedures (see Materials and Methods). standard, Standard procedure; euc clust, Euclidean clustering; non-euc clust, non-Euclidean clustering; covar norm, covariance normalization; var norm, variance normalization.

ination performance as a function of flash strength and time offset (Fig. 2). Figure $5 A$ shows, for a single RGC, the dependence of discrimination performance on flash strength for the two time offsets illustrated in Figure 4. Figure $5 B$ shows the discrimination surface across all time offsets and flash strengths probed, and Figure $5 C$ shows the fit of Equation 1 to this surface. The discrimination contour (probability correct, 0.84) is shown superim- 
posed on the fit in Figure $5 C$ and is replotted in Figure $5 D$ along with linearly interpolated values from the surface in Figure $5 B$. Finally, Figure $5 E$ shows the contours for all cells simultaneously recorded in this retina. Although contours exhibit a variety of shapes, a subset of cells provided the highest sensitivity across most of the range of flash strengths and time offsets probed.

To determine whether the discrimination procedure accurately indicated the detection and timing fidelity of RGC signals, its performance was compared with that of four more elaborate discrimination procedures (see Materials and Methods). Each of these yielded similar results (Fig. $5 F$ ), suggesting that the standard procedure was nearly optimal.

\section{Discrimination using the pool of rods within an RGC receptive field}

The flash strengths and time offsets that yielded near-threshold discrimination performance for RGCs were substantially smaller than those for rods, as can be seen by comparing the contours in Figures $3 E$ and $5 E$. This is expected because salamander RGCs pool inputs from hundreds of rods, and pooling should permit discrimination of weaker flashes separated by shorter times. Thus, to determine whether the fidelity of rod signals limits RGC performance, RGC performance was compared with that of the entire pool of rods within its receptive field. It was impractical to collect data from hundreds of rods at the low flash strengths required to perform this comparison directly, so a model was developed to simulate the responses of the pool of rods in the RGC receptive field.

The model was based on known properties of rods (Baylor et al., 1979b, 1984) and data such as those shown in Figures 1-3. For any given flash strength and time offset, the model provides a description of the distribution across trials of the correlation between the response of a single rod and the discriminant (Fig. 2). Assuming that the number of absorbed photons obeys Poisson statistics, and that dark noise and variability in the single-photon response are independent, Gaussian, and additive, the probability density $P_{c}$ for the correlation $c$ between a rod signal and the discriminant in a single trial is given by the following:

$$
P_{c}=\sum_{n} \frac{\bar{n}^{n} \exp (-\bar{n})}{n !} \frac{\exp \left[-(c-n \bar{c})^{2} /\left(\sigma_{d}^{2}+n \sigma_{c}^{2}\right)\right]}{\sqrt{2 \pi\left(\sigma_{d}^{2}+n \sigma_{c}^{2}\right)}} .
$$

The first term describes the probability that $n$ photons are absorbed given the mean $\bar{n}$. The second term describes the probability of obtaining a correlation $c$ given $n$ absorbed photons, a mean single-photon response $\bar{c}$, and two sources of rod noise: (1) dark noise with an SD of $\sigma_{d}$; and (2) variability in the singlephoton response, with a SD of $\sigma_{c}$. The parameter $\sigma_{d}$ approximates the combined effect of two sources of rod dark noise: spontaneous activation of rhodopsin and continuous fluctuations in rod current. This approximation did not compromise the ability of the model to capture rod discrimination performance, as described below, probably because the continuous noise dominates in salamander rods.

The parameters of the model were estimated for individual rods using data such as those in Figure 2 (see Materials and Methods). Figure 6, $A$ and $B$, shows two of the measured correlation distributions from Figure $2 B$ along with model fits.

To verify that the model provided an accurate summary of the detection and timing sensitivity of single rods, discrimination performance based on the entire rod signal waveform (as in Fig. 2 ) was compared with performance based on simulations gener-
A.

B.

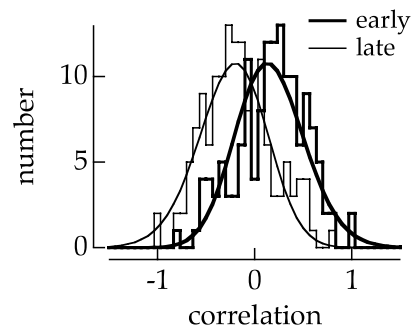

C.
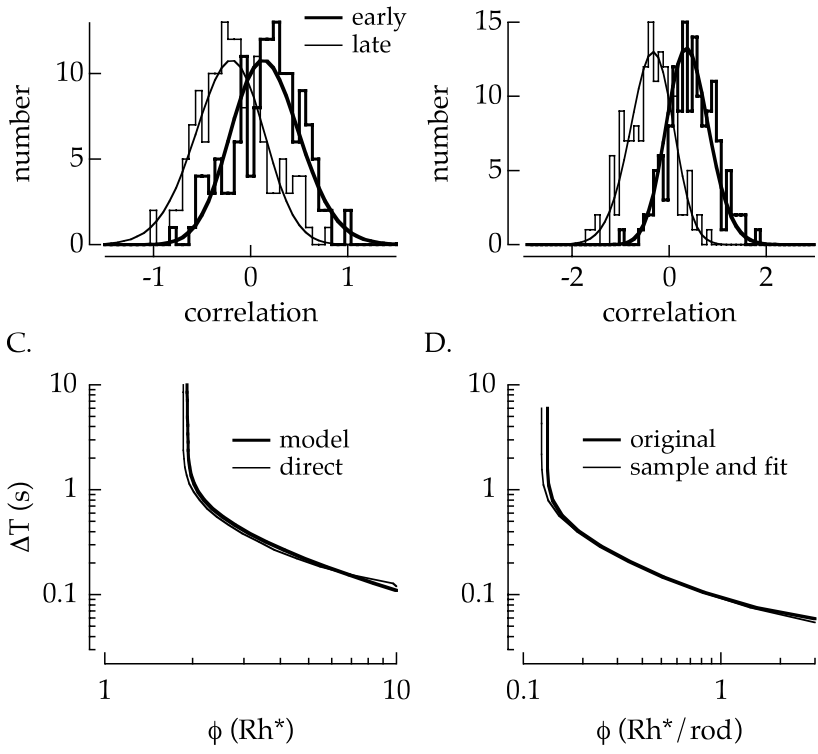

Figure 6. Model for rod discrimination signals. A, Measured (stepped curves) and fitted (smooth curves) correlation distributions for rod responses to a flash with strength $0.9 \mathrm{Rh}^{*}$ and time offset of $5 \mathrm{sec}$. $B$, Measured and fitted correlation distributions for a flash with strength 3.6 $\mathrm{Rh}^{*}$ and time offset of $0.2 \mathrm{sec}$. C, Discrimination contour obtained from the rod responses (thin line) or from the fits to the correlation distribution (thick line). D, Control for errors extrapolating model parameters to low flash strengths (see Materials and Methods). Discrimination contours from the original model (thick line) and from the fit to samples generated by the model (thin line).

ated with the model. Discrimination contours using the two approaches were similar (Fig. 6C). Simulations of the extrapolation involved in estimating the discrimination performance of rods to the lower flash strengths used in probing RGC sensitivity confirmed its accuracy (Fig. 6D) (see Materials and Methods).

\section{Comparison of rod and RGC detection sensitivity and temporal resolution}

To determine how faithfully RGCs signaled photon absorption, the discrimination performance of each RGC was compared with the performance expected from a noiseless summation of rod inputs weighted by the $\mathrm{RGC}$ receptive field profile (Fig. 7A). Figure $7 B$ shows the receptive field of one RGC measured using a white noise stimulus, and the superimposed ellipse shows the 2 SD boundary of an elliptical Gaussian fit (see Materials and Methods). The maximum discrimination performance expected from a linear combination of rod signals within this perimeter was computed as follows (Fig. 7A). A discrimination signal $c_{n}$ was generated for each rod using the model above (Eq. 3). The signal from each rod was weighted by the receptive field profile $w_{n}$ at its location, and the signals from all rods were summed. The sign of this sum was used to infer the time of the flash (early or late). This was repeated many times at each flash strength and time offset tested, and the fraction of correct discriminations was measured.

Because correlation with the discriminant is a linear operation, the above procedure is equivalent to computing the weighted sum of the response time course of all rods and correlating the resulting waveform with the discriminant. Given the weights (receptive field profile) used, this should provide discrimination performance as high as any other linear discrimination procedure, because all rods are nominally identical and the discriminant waveform provided near-optimal performance for each rod (Fig. $3 F$ ). Nonlinear pooling of rod signals was not 
A.

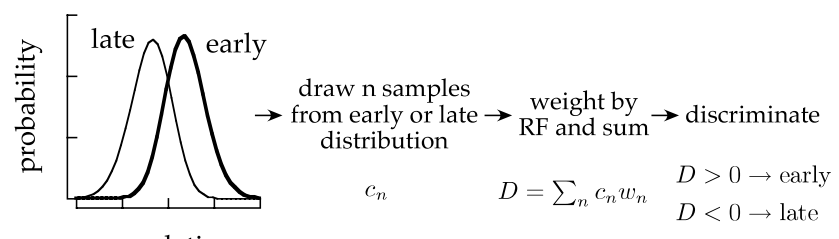

B.
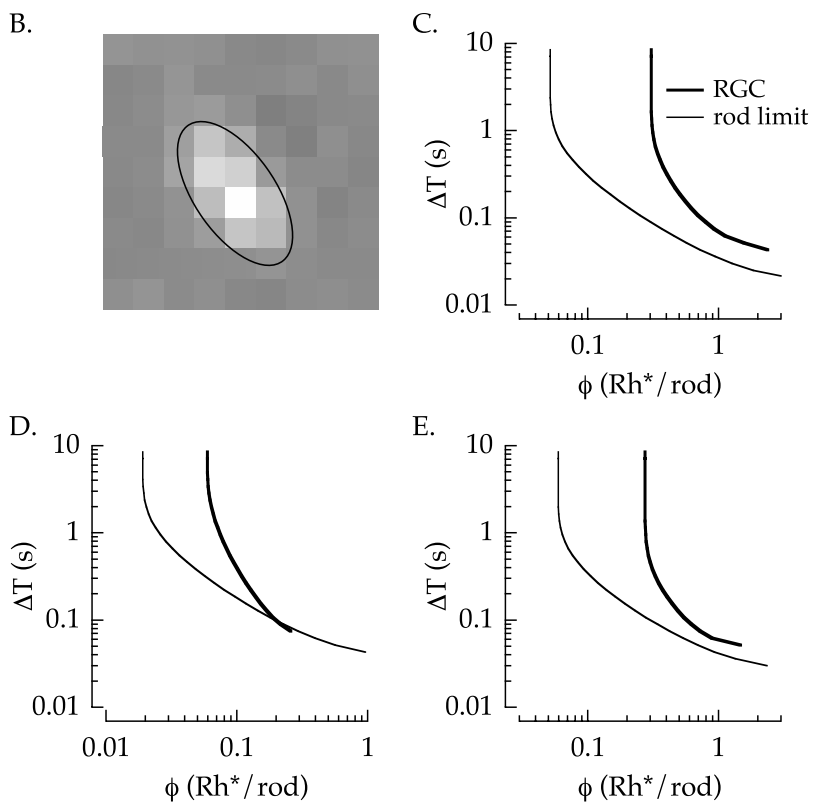

Figure 7. Comparison of RGC discrimination performance with rod limit. A, Schematic of model used to estimate sensitivity of rod pool. Samples drawn from the distribution of discrimination signals for early and late flashes were weighted by the receptive field (RF) profile and summed. Discrimination was based on the sign of the difference of these summed signals. $B$, $\mathrm{RGC}$ receptive field measured with white noise stimulation. Ellipse shows the $2 \mathrm{SD}$ contour of an elliptical Gaussian fit to the receptive field (see Materials and Methods). ( $-E$, Discrimination contours for individual RGCs from three retinas (thick lines) and corresponding rod limits (thin lines). The estimated number of rods within the 2 SD boundary for each RGC was 630,780 , and 450. Estimated detection thresholds (vertical asymptote of discrimination curve) were 0.30 , 0.06 , and $0.27 \mathrm{Rh}^{*} / \mathrm{rod}$.

considered because (1) electrical coupling between rods effectively enforces linear pooling, (2) the dominance of continuous noise limits the potential benefits of nonlinear pooling, and (3) nonlinear signal transfer at the rod-bipolar synapse has not been observed in salamander (see Discussion).

Figure $7 C$ (thin line) shows the discrimination contour obtained from the rod pool simulation. This indicates the performance that would be expected from a noiseless weighted sum of rod inputs. The discrimination contour obtained from the RGC is also shown (thick line). At long time offsets, for which the task effectively amounts to flash detection, the large horizontal separation between the curves (a factor of 6) indicates that RGC performance fell far short of the performance of the rod pool. Thus, the detection threshold for the RGC was significantly higher than that of an ideal observer of the rod signals within its receptive field.

At smaller time offsets, for which discrimination performance depends on both time offset and flash strength, the performance of the RGC more closely approached the rod limit. For example, at a time offset of $60 \mathrm{msec}$, the flash strength required for criterion performance $\left(1.1 \mathrm{Rh}^{\star} / \mathrm{rod}\right)$ was within a factor of 2 of the limit set by the rods $\left(0.6 \mathrm{Rh}^{\star} / \mathrm{rod}\right)$. Figure $7, D$ and $E$, shows discrimina-
A.

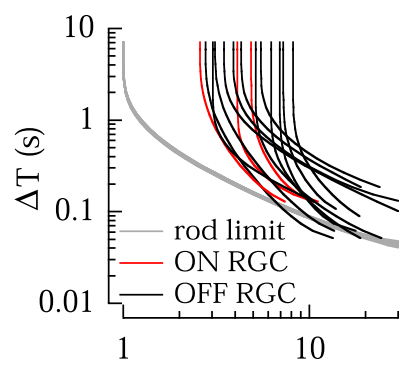

C.

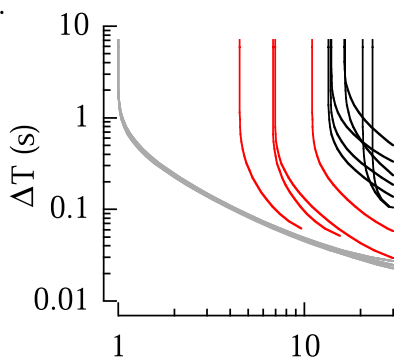

E.

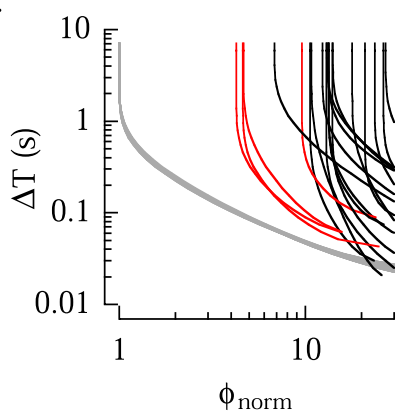

B.

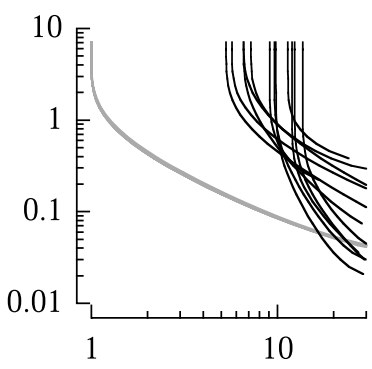

D.

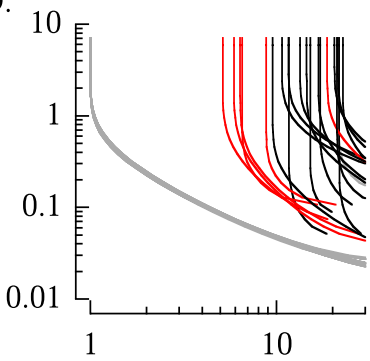

F.

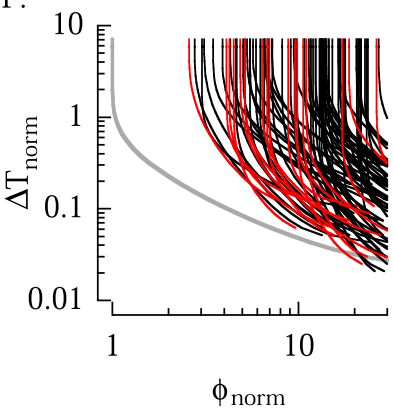

Figure 8. Summary of $R G C$ sensitivity relative to rod limit. $A-E$, Each panel shows discrimination contours for all ON (red) and OFF (black) RGCs recorded simultaneously in one retina and the corresponding rod limit. Rod and RGC discrimination contours were shifted along the flash strength axis to align the rod limit contours. F, Collected results from all 85 recorded cells (17 ON and $680 \mathrm{FF})$. Data from recordings in $1 \mathrm{~mm} \mathrm{Ca}^{2+}(A, B)$ were shifted along the time offset axis to align the rod limit contours with those obtained in $2 \mathrm{~mm} \mathrm{Ca}^{2+}(C-E)$.

tion contours and rod limit curves for selected RGCs from two other retinas. As with the first cell, the discrimination threshold far exceeded the rod limits at long time offsets but reached or approached the rod limit at shorter time offsets.

Figure $8 \mathrm{~A}$ shows pooled results for all cells recorded in one retina. Because the receptive field size of each RGC was different, the rod limit curve for each RGC was different. To represent discrimination contours from multiple cells on a single graph, the discrimination contour for each RGC and its corresponding rod limit curve were shifted together along the flash strength axis, by an amount required to bring the rod limit curves for all cells into register. The leftmost cluster of curves (gray) represents the shifted rod limits for all 15 RGCs recorded. The registration of these curves indicates that the dependence of the rod limit on receptive field size was equivalent to a scaling of flash strength. The red and black curves show the shifted discrimination contours for ON and OFF RGCs, identified by the sign of the dominant component of the response time course measured with white noise stimulation (see Materials and Methods). For all cells, the flash strength required for criterion performance at long time offsets, i.e., detection threshold, was 3-10 times higher than the rod limit. For some cells, a similar discrepancy persisted at short 
time offsets. For others, the discrimination contour approached the rod limit at time offsets of 50-150 msec, a value approximately one order of magnitude smaller than the rod integration time.

Figure $8 B-E$ shows results for the remaining four retinas examined, in the same format, and Figure $8 \mathrm{~F}$ shows pooled results from all 85 cells from all five retinas. As with the first retina, discrimination at low flash strengths and long time offsets (detection sensitivity) was far from the rod limits, but, for a subset of cells in each preparation, discrimination at high flash strengths and short time offsets (temporal resolution) approached the rod limits. ON cells (red curves) exhibited significantly lower detection thresholds than OFF cells (black curves) in three of four retinas in which ON cells were observed; ON and OFF cells had similar detection thresholds in the other retina. A more systematic examination of the performance of different cell types was not possible because an extensive analysis failed to reveal a functional cell type classification that was consistent across retinas. In summary, although detection of weak flashes by RGCs never approached the limits imposed by rod noise, the temporal discrimination of clearly detectable flashes did for some RGCs.

\section{Limits to performance in the retinal circuitry}

What causes RGC discrimination performance to fall short of the rod pool limit for low flash strengths and long time offsets but approach the rod pool limits for high flash strengths and short time offsets? Several aspects of cellular and synaptic computations in the retina could limit discrimination. Post-rod noise could obscure the rod signals. Thresholding or temporal filtering could compromise sensitivity but decrease output noise or response latency. Technical limitations in the experimental and analysis procedure could also contribute to the apparent failure of the RGCs to reach the rod limit. Each of these possibilities is considered below.

\section{Technical limitations}

Certain experimental artifacts and incorrect assumptions can be excluded as the main limit to performance. Light calibration error or photopigment bleaching, incorrect estimates of rod collecting area or RGC receptive field size, the presence of an antagonistic surround, and the possibility that RGCs collect inputs from only a subset of rods within the receptive field (Brown et al., 2000) would all scale the effective flash strength. Such a scaling would result in an apparent shift of discrimination contours along the abscissa, which could explain the observed discrimination contours of some RGCs. However, discrimination contours of cells such as those in Figure 7 were not shifted copies of the rod limit: a larger shift would be required to bring the RGC and rod limit contours into correspondence at low flash strengths than at high flash strengths. Thus, these technical factors cannot fully explain the relationship between RGC sensitivity and the limits imposed by rods.

\section{Added noise}

Noise in the retinal circuitry could limit the performance of RGCs. Figure $9 A$ shows rod pool discrimination contours with and without Gaussian noise added to the pooled rod signal amplitude. The noise was equivalent to increasing the dark noise $\left(\sigma_{d}\right.$ in Eq. 3) of each rod by a factor of 4 . Added noise results in a rightward shift of the entire discrimination contour and thus alone cannot account for the differences between RGC performance and the rod limit. This does not exclude the possibility of non-additive noise sources, e.g., stimulus-dependent noise that limits detection of weak flashes but is smaller for stronger flashes.
A.

B.
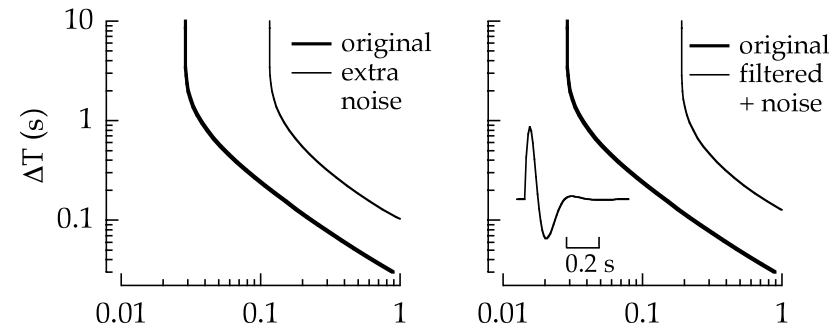

C.

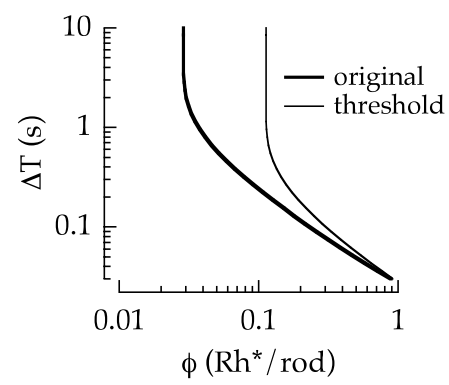

D.

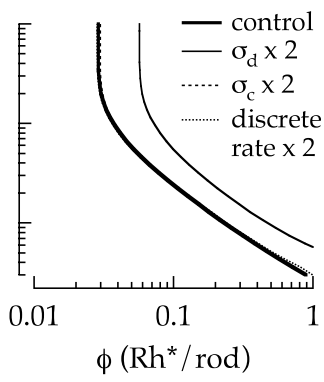

Figure 9. Sensitivity of rod limit to increased noise and limits in retinal processing. $A$, Rod limit contours calculated for a $160-\mu \mathrm{m}$-diameter receptive field with (thin trace) and without (thick trace) additive noise with an SD of $4 \sigma_{d}$ (see Eq. 3). B, Rod limit contours with (thin trace) and without (thick trace) high-pass filtering and added noise with SD of $0.2 \sigma_{d}$. The impulse response of the filter is indicated in the inset. $C$, Rod limit contours with (thin trace) and without (thick trace) a threshold that eliminated rod pool responses smaller than $0.08 \mathrm{Rh}^{*} / \mathrm{rod} . \mathrm{D}$, Sensitivity of rod limit contours to increases in each source of rod noise.

\section{Temporal filtering}

Retinal processing attenuates low-frequency components of the rod response (Schnapf and Copenhagen, 1982; Armstrong-Gold and Rieke, 2003), causing RGC responses to be briefer than those of the rods (Figs. 1, 4). A combination of temporal filtering and added noise in the retinal circuitry could limit RGC performance. To test for this possibility, before discrimination, each rod response was convolved with a filter (Fig. 9B, inset) that approximately accounted for the discrepancy between rod and RGC integration times near detection threshold ( $\sim 1$ vs $\sim 0.3 \mathrm{sec}$ ). Filtering alone should not affect performance because an appropriate discrimination procedure could undo the filtering (e.g., a high-pass filter can be undone by a complementary low-pass filter). Thus, noise was added after filtering, as in Figure 9A, to make the attenuation of low frequencies effectively irreversible; the added noise was 20 times smaller than that in Figure 9A. The combination of filtering and additive noise shifted the entire discrimination contour to the right (Fig. 9B) but did not preferentially decrease sensitivity at long time offsets. This suggests that discrimination at all time offsets relied on similar temporal frequencies of the rod response. Indeed, eliminating all frequencies $<0.5 \mathrm{~Hz}$ and $>3 \mathrm{~Hz}$ had little impact on the discrimination contour (data not shown). Thus, a combination of high-pass filtering and added noise could not account for the difference between RGC sensitivity and the rod limit.

\section{Thresholding}

Thresholding in the retinal circuitry could selectively suppress small rod signals essential for detection but retain larger rod signals that mediate discrimination at shorter time offsets. Figure $9 C$ shows discrimination contours obtained with and without a threshold that eliminated rod pool signals for flashes weaker than $0.08 \mathrm{Rh}^{\star} / \mathrm{rod}$. This simulates the achievable limit of performance 
in the case of a threshold in the retinal circuitry after pooling of rod signals. The thresholded rod pool exhibits a detection sensitivity much lower than that of the original rod pool but exhibits sensitivity approaching the rod pool limit at higher flash strengths and lower time offsets. This resembles the behavior of the most sensitive RGCs examined. The supralinear dependence of the RGC response on flash strength (Fig. 4) is consistent with such a threshold; indeed, in most RGCs, flashes producing $<0.1$ $\mathrm{Rh}^{\star} / \mathrm{rod}$ did not produce an identifiable response (data not shown). Such a threshold would compromise sensitivity at the lowest light levels probed but produce a low dark firing rate and nearly deterministic response (see Discussion).

\section{Continuous noise limits the fidelity of rod signals}

The above results show that RGC stimulus discrimination at short time offsets and flash strengths near $1 \mathrm{Rh}^{\star} /$ rod approaches the limits set by rod noise. Three sources of noise contribute to rod signals: discrete noise events attributable to spontaneous activation of rhodopsin (Baylor et al., 1980), continuous noise attributable to spontaneous activation of phosphodiesterase (PDE) (Baylor et al., 1980; Rieke and Baylor, 1996), and variations in the single-photon response (Rieke and Baylor, 1998a; Whitlock and Lamb, 1999; Field and Rieke, 2002). The impact of each noise source was evaluated by simulations of rod responses in which one noise source was increased and the effect on the discrimination contour was examined.

Figure $9 D$ compares the control discrimination contour with contours in which different sources of rod noise were increased. The simulations were made using the collection of rods within a typical RGC receptive field. Response fluctuations were increased by doubling $\sigma_{c}$ in Equation 3. Noise attributable to spontaneous activation of rhodopsin was increased by adding single-photon responses to the measured rod responses at random times and at a rate equal to the measured physiological rate of $0.03 \mathrm{~Hz}$ (Baylor et al., 1980; Vu et al., 1997). Both continuous dark noise and noise attributable to spontaneous activation of rhodopsin were increased by doubling $\sigma_{d}$ in Equation 3. Any changes in sensitivity observed with increased $\sigma_{d}$ but not observed with increased spontaneous activation noise alone may be attributed to continuous noise. Figure $9 D$ shows that increasing the spontaneous activation noise or the response fluctuations had little effect on the rod discrimination contour, whereas increasing both spontaneous activation noise and continuous noise substantially reduced sensitivity. Together, these observations indicate that the sensitivity of rod signals was limited almost entirely by continuous noise.

\section{Discussion}

We compared RGC sensitivity with the limit imposed by rod noise using a two-alternative forced-choice task. For all RGCs examined, detection threshold (stimulus discriminability at low flash strengths and large time offsets) was significantly higher than predicted from an ideal observer of rod signals within the RGC receptive field. For many RGCs, however, temporal resolution (discrimination at higher flash strengths and smaller time offsets) approached the rod limit, indicating that, in these conditions, the retinal circuitry faithfully preserved stimulus information represented in the rods. Thus, for some RGCs and some light levels, synaptic and posttransduction cellular noise did not substantially contaminate retinal signals. Furthermore, the process of spike generation required to transmit retinal signals to the brain did not impose a bottleneck on temporal resolution.

\section{Limits to detection sensitivity and timing discrimination in the retina}

The relationship between RGC and rod pool discrimination thresholds across flash strengths could not be explained by additive noise or high-pass filtering in the retina, which would cause RGCs to fall short of the rod limit at all flash strengths (Fig. 9A,B). This relationship, however, could be explained by a threshold in the retinal circuitry that eliminates small responses (Fig. 9C).

In the mouse retina, a threshold-like nonlinearity in signal transfer from rods to rod bipolar cells serves to transmit rod single-photon responses while rejecting noise (Field and Rieke, 2002; Sampath and Rieke, 2004). However, a threshold at the rod-to-bipolar synapse is unlikely to explain the present results. First, such a threshold is not observed in rod-to-bipolar signal transfer in salamander (Armstrong-Gold and Rieke, 2003). Second, strong electrical coupling between salamander rods (Attwell et al., 1984) causes the single-photon response generated in one rod outer segment to spread to 10-20 neighboring rod inner segments. The effective summation of many rod signals before the rod-bipolar synapse makes separation of single-photon responses and continuous noise difficult or impossible. Third, a threshold acting on the signals of individual rods would have a similar effect on sensitivity for all flash strengths well below 1 $\mathrm{Rh}^{\star} / \mathrm{rod}$, because the likelihood of a rod absorbing two photons at these flash strengths is low. This is inconsistent with the shapes of the contours shown in Figure 7. Thus, to explain the discrepancies between RGC sensitivity and the rod limit, thresholding must occur after the pooling of rod signals. Candidate mechanisms include rectification at the bipolar-RGC synapse and in RGC spike generation (Dhingra and Smith, 2004).

Salamander RGCs fire at rates below $1 \mathrm{~Hz}$ in darkness, much lower than the $10-20 \mathrm{~Hz}$ rate of spontaneous activation of rhodopsin in the rods within the RGC receptive field. This is consistent with a threshold in the retinal circuitry, again suggesting that the sensitivity of salamander RGCs at low light levels is limited more by failure to respond than by noise. Indeed, more than a few spikes in 100-200 msec almost invariably indicates the presence of a stimulus. Similar nearly deterministic responses have been described in toad RGCs (Copenhagen et al., 1987).

At flash strengths near $1 \mathrm{Rh}^{\star} / \mathrm{rod}$, the responses of many RGCs permitted reliable discrimination of flashes separated by $<100 \mathrm{msec}, \sim 5 \%$ of the integration time of the rod light response. This is one of several examples in which acuity exceeds naive expectations based on the properties of receptor signals. For example, humans can detect spatial displacements smaller than the spacing between foveal cones provided the stimuli cover multiple cones (Westheimer, 1981). Chromatic acuity is another example: the ability to discriminate changes in wavelength of monochromatic lights is much finer than the width of cone spectral sensitivity curves (Mollon et al., 1992). In both cases, receptor noise poses a fundamental limit to acuity, and acuity finer than the nominal spatial or wavelength resolution can be explained by a high signal-to-noise ratio, sometimes achieved by pooling of inputs from multiple receptors. The correspondence between spatial and chromatic acuity and receptor noise in humans has not been explored in detail, but, in the fly visual system, receptor signal and noise can explain the ability of motion-sensitive neurons to detect movements smaller than the spacing between photoreceptors (Bialek et al., 1991). Similarly, the present results show that RGCs can achieve timing discrimination much finer than the rod integration time and that this discrimination approaches the limit set by noise in the rods. 


\section{Possible sources of error}

A major focus of the present study was measurement of rod and RGC sensitivity with greater precision than had been achieved previously, to assess how closely RGC signaling approaches the limits set by the rods. The two-alternative classification approach was adopted because, unlike approaches using richer stimulus sets, the sensitivity of rods and RGCs could be assessed with near-optimal classification procedures that involved minimal assumptions about how visual signals are represented. Even so, several potential sources of error remain as a result of finite data and assumptions made in the analysis.

It was assumed that every rod within the RGC receptive field provides input to the RGC, with a strength determined by a Gaussian spatial profile. Although this is consistent with findings in cat retina (Freed and Sterling, 1988; Sterling et al., 1988), receptive field microstructure inconsistent with these assumptions has been documented in rabbit retina (Brown et al., 2000). Such microstructure could reduce RGC sensitivity by suppressing or excluding informative rod signals. It was also assumed that the receptive field surround is negligible. Although the surround is weak at the light levels probed (Copenhagen et al., 1987), in some cases, surrounds were observed at the higher light levels used to measure receptive fields. The surround, if active during detection threshold measurements, could reduce RGC sensitivity by suppressing the center signal and adding independent noise. Either receptive field microstructure or surrounds would shift the RGC discrimination contour along the abscissa away from the rod pool limit. Because many RGCs approached the rod pool limit for small time shifts, these effects are probably small. Finally, because the rod pool limit scales inversely with the square root of the number of rods in the receptive field, the results are relatively insensitive to misestimates of effective receptive field size.

The control analyses presented indicate that a simple discrimination procedure provided near-optimal discrimination within the limits of the data. Thus, the procedure approximates ideal observer analysis, which has been used to infer the efficiency of behavioral discriminations given known optical and neural constraints (Geisler, 1989). However, the control analyses presented here do not exclude the possibility that other procedures could extract more stimulus information from neural signals or that larger data sets could permit more reliable estimation of the statistics of neural responses and thus more accurate discrimination. The latter limitation is particularly acute for RGCs, in which response nonlinearities precluded combining data across flash strengths in choosing a discriminant.

Finally, the data were obtained from isolated rods and from RGCs in isolated retinas. It is possible that these experimental procedures compromise RGC sensitivity more than rod sensitivity, specifically at lower flash strengths.

\section{Impact of rod noise sources on retinal sensitivity}

The present findings show that, at high flash strengths and short time offsets, noise in rod photocurrents limits the response fidelity of salamander RGCs. The three main sources of noise in rod outer segments are as follows: (1) continuous fluctuations attributable to spontaneous PDE activation (Baylor et al., 1980; Rieke and Baylor, 1996); (2) discrete noise events attributable to spontaneous activation of rhodopsin (Baylor et al., 1980); and (3) variation in the single-photon response attributable to fluctuations in rhodopsin activity (Rieke and Baylor, 1998a; Whitlock and Lamb, 1999; Field and Rieke, 2002). The relative importance of these noise sources for visual performance has not been established. Furthermore, the limiting noise source may vary with species; in salamander rods, continuous noise accounts for a larger fraction of the dark noise than it does in toad (Baylor et al., 1980) or primate rods (Baylor et al., 1984).

Previous comparisons of rod noise with behavioral sensitivity have emphasized the role of spontaneous activation of rhodopsin in limiting absolute visual sensitivity. Psychophysical studies have established that human observers can detect the absorption of 5-10 photons in a pool of 300-500 rods (Hecht et al., 1942; Sakitt, 1972). Under the assumption that detection is limited by spontaneous activation of rhodopsin, these studies provided estimates of the event rate that are comparable with experimentally measured rate in primate rods (Baylor et al., 1984). However, this comparison is accurate only to within a factor of 3-5 because of uncertainties in both rod and behavioral measurements (Barlow, 1977; Schneeweis and Schnapf, 2000). In amphibians, the temperature dependence of behavioral threshold has been compared with expectations from measurements of the temperature dependence of the spontaneous activation of rhodopsin (Aho et al., 1987, 1993). However, substantial differences between species make it difficult to draw a universal conclusion about whether spontaneous activation of rhodopsin limits behavioral threshold (Aho et al., 1993).

RGC sensitivity has also been compared with expectations based on spontaneous activation of rhodopsin in rods. Recordings from cat retina have suggested that maintained firing in dark-adapted RGCs is produced by spontaneous activation of rhodopsin in rods (Barlow et al., 1971; Mastronarde, 1983a,b). This maintained firing obscures the signal generated by dim flashes, apparently providing the main limit to absolute sensitivity (Barlow et al., 1971), unlike the present findings. A potential caveat to interpretation of the cat studies is that the receptive fields of the specific RGCs recorded were not measured, precluding a direct comparison between the maintained firing rate and the pool of rods providing input to the RGC. Alternatively, the limiting sources of rod noise may differ in cats and salamanders; little is known about noise in cat rods.

The most unambiguous comparison of RGC sensitivity with rod noise used frequency of seeing analysis with the responses of toad RGCs (Copenhagen et al., 1987). In the most sensitive RGCs, detection was limited by an equivalent noise within a factor of 2 or 3 of that expected from spontaneous activation of rhodopsin. Together with the low spontaneous firing rate of these cells, this suggests a threshold positioned slightly higher than the mean input from spontaneous activation of rhodopsin. In salamander rods, the relative amplitude of continuous noise is several-fold higher than in toad rods (F. Rieke, unpublished observations). Consequently, continuous noise rather than spontaneous activation of rhodopsin or response fluctuations imposes the dominant limit on sensitivity (Fig. 9D). Thus, in the salamander, unlike the toad, it is not possible for RGC sensitivity to approach the limits imposed by spontaneous activation of rhodopsin using an appropriate response threshold. Instead, the response threshold is apparently so high that it ultimately limits detection sensitivity (Fig. 9C). However, in a subset of RGCs, discrimination performance approached the rod limit for high flash strengths $\left(1 \mathrm{Rh}^{\star} / \mathrm{rod}\right)$ and short temporal offsets (100 $\mathrm{msec})$. In these conditions, continuous noise is the dominant limit on the fidelity of retinal signals transmitted to the brain.

\section{References}

Aho AC, Donner K, Hyden C, Reuter T, Orlov OYu (1987) Retinal noise, the performance of retinal ganglion cells, and visual sensitivity in the dark-adapted frog. J Opt Soc Am A 4:2321-2329. 
Aho AC, Donner K, Hyden C, Larsen LO, Reuter T (1988) Low retinal noise in animals with low body temperature allows high visual sensitivity. Nature 334:348-350.

Aho AC, Donner K, Reuter T (1993) Retinal origins of the temperature effect on absolute visual sensitivity in frogs. J Physiol (Lond) 463:501-521.

Armstrong-Gold CE, Rieke F (2003) Bandpass filtering at the rod to secondorder cell synapse in salamander (Ambystoma tigrinum) retina. J Neurosci 23:3796-3806.

Attwell D, Wilson M, Wu SM (1984) A quantitative analysis of interactions between photoreceptors in the salamander (Ambystoma) retina. J Physiol (Lond) 352:703-737.

Barlow HB (1977) Retinal and central factors in human vision limited by noise. In: Vertebrate photoreception (Barlow HB, Fatt P, eds), pp 337351. New York: Academic.

Barlow HB, Levick WR, Yoon M (1971) Responses to single quanta of light in retinal ganglion cells of the cat. Vision Res Suppl 3:87-101.

Baylor DA, Lamb TD, Yau KW (1979a) The membrane current of single rod outer segments. J Physiol (Lond) 288:589-611.

Baylor DA, Lamb TD, Yau KW (1979b) Responses of retinal rods to single photons. J Physiol (Lond) 288:613-634.

Baylor DA, Matthews G, Yau KW (1980) Two components of electrical dark noise in toad retinal rod outer segments. J Physiol (Lond) 309:591-621.

Baylor DA, Nunn BJ, Schnapf JL (1984) The photocurrent, noise and spectral sensitivity of rods of the monkey Macaca fascicularis. J Physiol (Lond) 357:575-607.

Bialek W, Rieke F, de Ruyter van Steveninck RR, Warland D (1991) Reading a neural code. Science 252:1854-1857.

Brown SP, He S, Masland RH (2000) Receptive field microstructure and dendritic geometry of retinal ganglion cells. Neuron 27:371-383.

Chichilnisky EJ (2001) A simple white noise analysis of neuronal light responses. Network 12:199-213.

Chichilnisky EJ, Baylor DA (1999) Receptive-field microstructure of blueyellow ganglion cells in primate retina. Nat Neurosci 2:889-893.

Chichilnisky EJ, Kalmar RS (2002) Functional asymmetries in ON and OFF ganglion cells of primate retina. J Neurosci 22:2737-2747.

Copenhagen DR, Donner K, Reuter T (1987) Ganglion cell performance at absolute threshold in toad retina: effects of dark events in rods. J Physiol (Lond) 393:667-680.

Dartnall HJA (1972) Photosensitivity. In: Handbook of sensory physiology, Vol VII/1, Photochemistry of vision, pp 122-145. New York: Springer.

Dhingra NK, Smith RG (2004) Spike generator limits efficiency of information transfer in a retinal ganglion cell. J Neurosci 24:2914-2922.

Donner K (1992) Noise and the absolute thresholds of cone and rod vision. Vision Res 32:853-866.

Duda RO, Hart PE (1973) Pattern classification and scene analysis, Chap 2. New York: Wiley.

Field GD, Rieke F (2002) Nonlinear signal transfer from mouse rods to bipolar cells and implications for visual sensitivity. Neuron 34:773-785.

Field GD, Sampath AP, Rieke F (2005) Retinal processing near absolute threshold: from behavior to mechanism. Annu Rev Physiol, in press.

Freed MA, Sterling P (1988) The ON-alpha ganglion cell of the cat retina and its presynaptic cell types. J Neurosci 8:2303-2320.

Geisler WS (1989) Sequential ideal-observer analysis of visual discriminations. Psychol Rev 96:267-314.
Harosi FI (1975) Absorption spectra and linear dichroism of some amphibian photoreceptors. J Gen Physiol 66:357-382.

Hecht S, Shlaer S, Pirenne MH (1942) Energy, quanta, and vision. J Gen Physiol 25:819-840

Hemila S, Lerber T, Donner K (1998) Noise-equivalent and signalequivalent visual summation of quantal events in space and time. Vis Neurosci 15:731-742.

Litke AM (1999) The retinal readout system: a status report. Nucl Instrum Methods Phys Res A 435:242-249.

Makino CL, Taylor WR, Baylor DA (1991) Rapid charge movements and photosensitivity of visual pigments in salamander rods and cones. J Physiol (Lond) 442:761-780.

Mastronarde DN (1983a) Correlated firing of cat retinal ganglion cells. I. Spontaneously active inputs to X- and Y-cells. J Neurophysiol 49:303-324.

Mastronarde DN (1983b) Correlated firing of cat retinal ganglion cells. II. Responses of X- and Y-cells to single quantal events. J Neurophysiol 49:325-349.

Meister M, Pine J, Baylor DA (1994) Multi-neuronal signals from the retina: acquisition and analysis. J Neurosci Methods 51:95-106.

Mollon JD, Astell S, Cavonius CR (1992) A reduction in stimulus duration can improve wavelength discriminations mediated by short-wave cones. Vision Res 32:745-755.

Rieke F, Baylor DA (1996) Molecular origin of continuous dark noise in rod photoreceptors. Biophys J 71:2553-2572.

Rieke F, Baylor DA (1998a) Origin of reproducibility in the responses of retinal rods to single photons. Biophys J 75:1836-1857.

Rieke F, Baylor DA (1998b) Single-photon detection by rod cells of the retina. Rev Mod Physics 70:1027-1036.

Sakitt B (1972) Counting every quantum. J Physiol (Lond) 223:131-150.

Sampath AP, Rieke F (2004) Selective transmission of single photon responses by saturation at the rod-to-rod bipolar synapse. Neuron 41:431-443.

Schnapf JL, Copenhagen DR (1982) Differences in the kinetics of rod and cone synaptic transmission. Nature 296:862-864.

Schneeweis DM, Schnapf JL (2000) Noise and light adaptation in rods of the macaque monkey. Vis Neurosci 17:659-666.

Sherry DM, Bui DD, Degrip WJ (1998) Identification and distribution of photoreceptor subtypes in the neotenic tiger salamander retina. Vis Neurosci 15:1175-1187.

Sterling P, Freed MA, Smith RG (1988) Architecture of rod and cone circuits to the on-beta ganglion cell. J Neurosci 8:623-642.

Teich MC, Prucnal PR, Vannucci G, Breton ME, McGill WJ (1982) Multiplication noise in the human visual system at threshold. 3. The role of non-Poisson quantum fluctuations. Biol Cybern 44:157-165.

Victor JD, Purpura KP (1997) Metric-space analysis of spike trains: theory, algorithms and application. Network 8:127-164.

Vu TQ, McCarthy ST, Owen WG (1997) Linear transduction of natural stimuli by dark-adapted and light-adapted rods of the salamander, Ambystoma tigrinum. J Physiol (Lond) 505:193-204.

Westheimer G (1981) Visual hyperacuity. Prog Sens Physiol 1:1-30.

Whitlock GG, Lamb TD (1999) Variability in the time course of single photon responses from toad rods: termination of rhodopsin's activity. Neuron 23:337-351. 